\title{
Magneto-Optical Kerr Effect Enhancement Methods for Nanostructures
}

\author{
D. H. Kim and Chun-Yeol You* \\ Department of Physics, Inha University, Incheon 402-751, Korea
}

(Received 27 November 2008, Received in final form 30 January 2009, Accepted 4 February 2009)

\begin{abstract}
Herein, the Magneto-Optical Kerr Effect (MOKE) signal enhancement in nanostructures in investigated. It is well known that the MOKE signals of ferromagnetic thin films are enhanced with an additional dielectric layer due to multiple reflections. The MOKE signal is modulated with the additional dielectric layer thickness and is at a maximum when reflectivity is at a minimum. This is not always true in the nanostructures due to the contribution from the non-magnetic substrate portion, especially when substrate reflectivity is minimized and the dependence of the additional dielectric layer thickness for the nanostructure is changed in the case of the continuous thin film. We showed that the MOKE signal for nanostructures could be enhanced with a properly designed, dielectric layer in addition to the anti-reflection coated substrates.
\end{abstract}

Keywords : Magneto-Optical Kerr Effect, magnetic nanostructure, nanodot, dielectric layer

\section{Introduction}

Magnetization dynamics of ferromagnetic nanostructures such as nanowires and nanodots is one of the most important subjects in current magnetism research. The magnetic dynamics in nanostructures are distinguished from thin films due to the confined geometry and corresponding boundary conditions [1]. Furthermore, the experimental measurements of the nanostructures have additional difficulties due to the small volume of the ferromagnetic body that causes small signals in most measurement methods. For example, the Magneto-Optical Kerr Effect (MOKE) is a well-developed measurement method for the magnetic dynamics in thin films given its ultrasensitivity, making it capable of resolving down to the sub-monolayer. In addition, with the pump-prove technique, its time resolving power can be down to the nanosecond [2]. However, its capability is limited to nanostructures because of its optical diffraction limits. The beam spot size is limited on the order of $\sim \lambda / N A$, where $N A(\sim 1)$ is the numerical aperture of the lens and $\lambda$ is the wavelength of the light, due to diffraction theory. Therefore, the beam spot size is limited to a few $100 \mathrm{~nm}$, which is somewhat larger than most interesting nanostructures. Furthermore, a well-focused beam will introduce inevitable heating,

*Corresponding author: Tel: $+82-32-860-7667$

Fax:+82-32-872-7562, e-mail: cyyou@inha.ac.kr altering the magnetic dynamics of the sample and even destroying it. There are many studies focused on overcoming the poor signal to noise ratio and increasing the MOKE signal for the nanostructures. For example, Cowburn et al. [3] and Beach et al. [4] took numerous averages from raw measurements to extract meaningful data. Therefore, MOKE has limited functionality for nanostructure measurement. Recently, another approach has reported that MOKE signals can be enhanced by using anti-reflection coated substrates for the nanostructure [5-12]. The underlying physics of signal enhancement with anti-reflection coated substrates is as follows: The simple physical meaning of the MOKE signal is the ratio between change in reflectivity due to the magneto-optical effect, and the reflectivity. Therefore, the reflective signal from the nonmagnetic portion will reduce the MOKE signal. For nanostructures, the majority of the beam falls into the nonmagnetic region and the substrate. The reflected beam from the substrate then contributes only to the reflectivity, so that it causes degradation of the MOKE signal. Such unwanted reflected beam could be suppressed by employing an anti-reflection coated substrate. As an example, when a substrate is employed whose reflectivity is $0.7 \%$ for a $100-\mathrm{nm}$ wide Fe nanowire, the MOKE signal is about $79 \%$ of its bulk value for a 1500 -nm radius beam [5].

In this study, we found that these MOKE signals from the nanostructures with the anti-reflection coated substrate 
are quite different from those of the continuous films as follows: First, the thicknesses of the ferromagnetic nanostructure dependence shows unexpected results; the MOKE signal from a $100-\mathrm{nm}$ nanowire with the anti-reflection coated substrate was larger than the continuous film, even when the ferromagnetic layer thickness was thinner than $6.0 \mathrm{~nm}$. Secondly, for the continuous thin film geometry, MOKE signal enhancement can be achieved simply by a dielectric layer coating $[13,14]$. Due to the multiple reflections and the reduction in reflectivity, MOKE signal enhancement can be established. It is well known that maximum signal enhancement is related to the anti-reflection conditions [15]. However, the discovered maximum MOKE signal conditions for the continuous film were not applicable to the nanostructures, especially in the case of the anti-reflection coated substrate.

\section{Magneto-Optical Kerr Effect for Nanostructures with Anti-reflection Coated Substrates}

MOKE manifests itself as the change in polarization of reflected light from the surface of ferromagnetic materials. The rigorous definition of MOKE requires a magnetooptical Fresnel reflection matrix [16,17]; however, the authors would like to introduce a simpler definition in this study in order to capture the essential physics. It must be noted that the numerical calculations in this work were performed with the medium boundary and propagation matrix method $[5,18]$, which is exact in a continuous film of the first order. The MOKE can be defined by the ratio between the change in reflectivity due to the change in the magnetization direction $(\Delta R)$ and reflectivity $R, \theta_{K}$ $=\Delta R / R$. Hereafter, for the sake of simplicity, this definition will be used in this study rather than a more rigorous definition.

The experimental situation for nanostructures such as nanowires or nanodots, typically involved a focused laser beam with a Gaussian profile where the detected MOKE signal is the sum of all the contributions from the substrate and the ferromagnetic nanostructures, as shown in Fig. 1 (a). While the substrate and nanostructure contribute to the reflectivity $(R)$, only ferromagnetic nanostructures contribute to the $\Delta R$. When the beam intensity profile, $I(x, y)$, is considered, the measured MOKE signal reads as:

$$
\Theta_{K}^{\mathrm{nano}}=\frac{\int I(x, y) \Delta R(x, y) d x d y}{\int I(x, y) R(x, y) d x d y} .
$$

The integrated reflected beam intensity from the sub- (a)

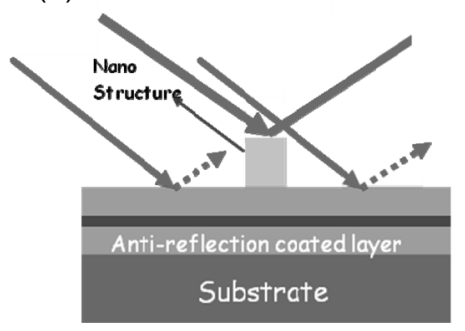

(b)

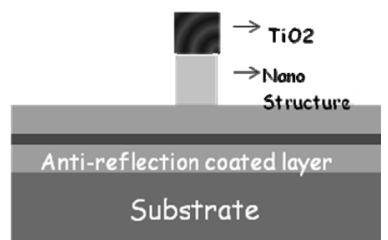

Fig. 1. (a) The concept of MOKE signal enhancement with anti-reflection coated substrates in the nanostructure. The antireflection coating minimizes contribution to the MOKE from the beam irradiated on the substrate. Dashed arrows illustrate the reflected beam of the substrate, indicating that their intensities are small. (b) An additional dielectric layer $\left(\mathrm{TiO}_{2}\right)$ is deposited on the nanostructure in order to achieve further MOKE signal enhancement.

strate $\left(R_{\text {sub }}\right)$ and the nanostructure itself, $\left(R_{\text {nano }}\right)$ and $\left(\Delta R_{\text {nano }}\right)$, can then be defined as the change in intensity due to the magneto-optical effect. Eq. (1) can then be rewritten as:

$$
\Theta_{K}^{\text {nano }}=\frac{\Delta R_{\text {nano }}}{R_{\text {sub }}+R_{\text {nano }}}
$$

This equation is the key idea in the enhancement of the MOKE signal by an anti-reflection coated substrate [5]. When $R_{\text {sub }}$ approaches zero because of the anti-reflection coating, the MOKE signal draws closer to its bulk value, $\Theta_{K}^{0}=\Delta R_{\text {nano }} / R_{\text {nano }}$. Dashed arrows illustrate the reflected beams from the anti-reflection coated substrate in order to represent small reflectivity, Fig. 1 (a).

\section{MOKE Signal of Nanodots}

Fig. 2 (a) shows calculated MOKE signals from various sizes of 10 -nm thick Fe nanodots on 4 substrates with different reflectivities, $\left(R_{\text {sub }}=0.7,2.1,6.0\right.$, and $\left.30 \%\right)$. Details of the calculations can be found in our previous work [5]. The problem space was divided by small cells in order to calculate the MOKE signal from the nanostructures. The medium boundary and propagation matrixes method was employed in order to calculate the reflected beam intensity with Gaussian profiles for $p$ - and $s$-polarizations [18]. The measured MOKE signal was then calculated by the sums of $R$ and $\Delta R$. The calculations in this study were performed under the following conditions: a wavelength of $632.8 \mathrm{~nm}$ (He-Ne laser); $\mathrm{Fe}$ is considered a magnetic material with $Q=0.0376+0.0066 i$ and $n=2.87$ $+3.36 i$, where $Q$ is the magneto-optic constant, the source of the MOKE $[19,20]$, and $n$ is the complex refractive index. Only the $s$-polarization wave with an incident 


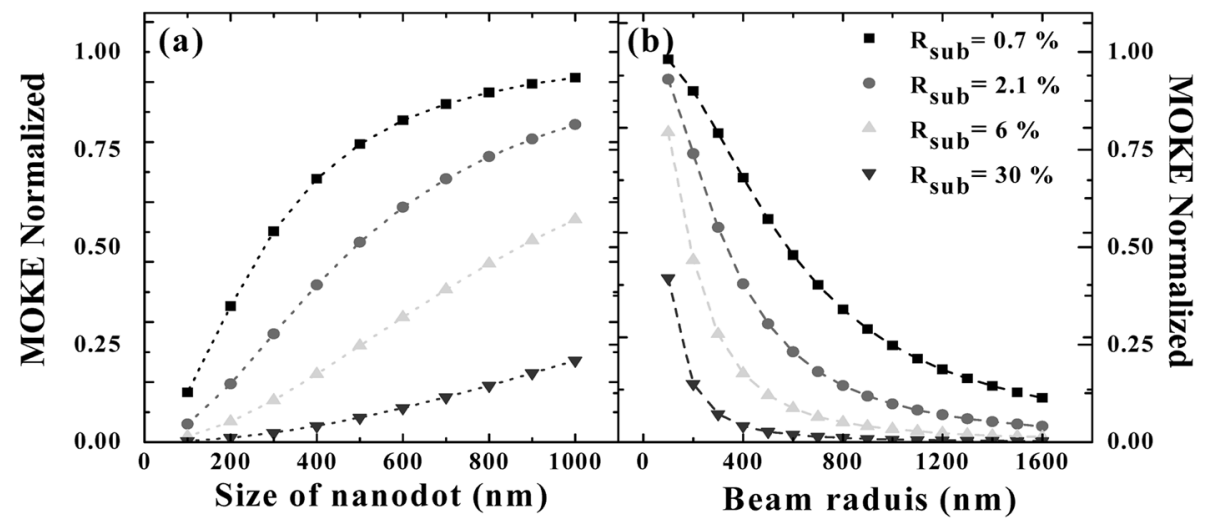

Fig. 2. Normalized MOKE signals plotted for: (a) various sizes of nanodots for a 1500 -nm radius Gaussian beam; (b) various beam radii for a $100-\mathrm{nm}$ radius nanodot for different substrate reflectivities $\left(R_{\text {sub }}=0.7,2.1,6.0,30 \%\right)$.

angle of $45^{\circ}$ was considered.

The radii of the $10-\mathrm{nm}$ thick Fe nanodots were varied from 100 to $1000 \mathrm{~nm}$, Fig. 2 (a). In this calculation, the laser beam radius was fixed at $1500 \mathrm{~nm}$, with its center assumed to be matched with the nanodot center. When the sizes of the nanodots decreased, the MOKE signals also decreased due to the smaller ferromagnetic area. However, the decrement of the MOKE signal was less significant for the smaller reflectivity substrate. For example, the MOKE signal for the $1000-\mathrm{nm}$ nanodot had a MOKE signal of $20 \%$ of its bulk value for $R_{\text {sub }}=30 \%$, while it was almost $97 \%$ for $R_{\text {sub }}=0.7 \%$. For 400 -nm nanodots, $6.0 \%(\sim 70 \%)$ of the bulk values for $R_{\text {sub }}=30 \%\left(R_{\text {sub }}=0.7\right.$ $\%)$ were obtained, respectively. Here, $70 \%$ of the bulk value indicates that a one-shot measurement can be performed, an important enhancement for the experiments. However, the enhancement is limited for 100-nm nanodots, $<1 \%(\sim 12 \%)$ of the bulk values for $R_{\mathrm{sub}}=30 \%\left(R_{\mathrm{sub}}=0.7\right.$ $\%$ ), as the signal was too small, even for the anti-reflection coated substrates. In Fig. 2 (a), the $R_{\text {sub }}=6.0$ and $2.1 \%$ cases were plotted together with intermediate results. Similar calculations for nanowires have been previously reported by our lab [5], thus only the nanodots are shown in this study. The general tendencies of nanowires and nanodots are similar, but it must be noted that the enhancement of a nanodot is poorer than a nanowire [5] due to its small area fraction. However, it still yields a significant enhancement for moderate sized nanodots. Fig. 2 (b) shows the dependence of the MOKE signal on the beam radius for the $10-\mathrm{nm}$ thick Fe nanodot with a $100-\mathrm{nm}$ radius. The beam radii were varied from 100 to $1600 \mathrm{~nm}$. When the beam radius was comparable to the dot size, the MOKE signal was close to the bulk value. In real experiments, such a small beam radius cannot be achieved due to the diffraction limit. As expected, the increment beam radius caused a poor MOKE signal. Even though the decrement of the MOKE signal for the $R_{\text {sub }}=0.7 \%$ was weaker than $R_{\text {sub }}=30 \%$, it is still too small. Therefore, greater improvement is required.

It should be emphasized here that calculations in this study are not exact results, even using the medium boundary and propagation matrixes method [18]. Since this method assumes a continuous film, it does not consider the finite size of the nanostructures. The rigorous vector diffraction theory and the near field optics framework must be applied for the nanostructure calculations. Therefore, the exact MOKE signal from a nanostructure must be calculated using complex numerical calculations such as the magneto-optical Finite Difference Time Domain (FDTD) [21,22]. However, in this case, a number of simple assumptions will be sufficient to distinguish the trend in the signal variations and capture the all-important physics and the correct, obtained tendencies.

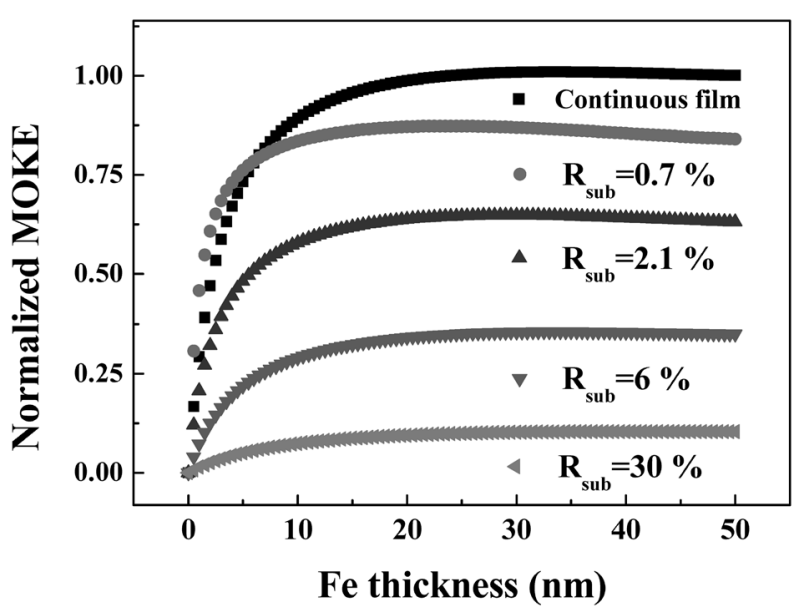

Fig. 3. Dependence of MOKE signals on Fe layer thickness for $100-\mathrm{nm}$ wide nanowires on various substrates. 


\section{Thickness Dependence of the MOKE Signal}

The MOKE signal depends on the thickness of the ferromagnetic layer as it is proportional to the amount of the magnetic substance. The thickness dependence of a metallic ferromagnetic material is not a simple monotonic increase function due to its large extinction coefficient in the visible light range [18]. The typical behavior of the thickness dependence of the MOKE signal is depicted in Fig. 3 for a continuous film. At a thickness thinner than $\sim 15 \mathrm{~nm}$, it increased rapidly and then slightly decreased to reach a final saturation value. This small oscillation is due to the multiple reflections inside the Fe layer. The thickness dependence of the MOKE for $100-\mathrm{nm}$ wide $\mathrm{Fe}$ nanowires with 4 kinds of substrates and a $1500-\mathrm{nm}$ radius beam was also plotted. All kinds of substrates showed similar trends, rapidly increasing in the thin region and saturating with a small overshoot. However, surprisingly, the MOKE signal from a 100 -nm wide nanowire had a larger MOKE signal compared to the continuous film when the thickness was thinner than $6.0 \mathrm{~nm}$. Additionally, the Fe layer thicknesses where the MOKE signals were maxima were also varied with substrate reflectivities.

\section{Effect of an Additional Dielectric Layer}

Enhancements of the MOKE signal in continuous films have been actively studied since the late $80 \mathrm{~s}$ due to its potential application in Magneto-Optical disks [23, 24]. It has been well known that an additional dielectric layer on top of the magnetic layer would greatly enhance the MOKE signal [6]. With the additional dielectric layer, 2 ingredients are helpful to enhance the MOKE. First, are the multiple reflections on the magnetic layer, and the second is reduction of reflectivity. Therefore, maximum enhancement is achieved when the dielectric layer thickness satisfies the anti-reflection conditions. When the dielectric layer thickness was varied, the MOKE signal oscillated as shown in Fig. 4, with a periodicity of $\sim 147$ $\mathrm{nm}$. The period was related to $\lambda / 2 n_{D} \cos \theta_{D} \sim 132 \mathrm{~nm}$, where the wavelength $(\lambda=632.8 \mathrm{~nm})$, the refractive index of the dielectric layer $\left(n_{D}=2.27\right)$, and the refractive angle $\left(\theta_{D}\right)$ was for $\mathrm{TiO}_{2}$. The MOKE signal from $10-\mathrm{nm}$ thick and $100 \mathrm{-nm}$ wide $\mathrm{Fe}$ nanowires on 4 different substrates was also performed. A beam radius of $1500 \mathrm{~nm}$ was used as shown in Fig. 1 (b). For $R_{\text {sub }}=30 \%$, the MOKE signal itself was small and the enhancement due to the dielectric layer not noticeable. However, a significant enhancement was observed in $R_{\text {sub }}=0.7 \%$. Herein, 2 findings need to be emphasized. At the maximum enhanced conditions,

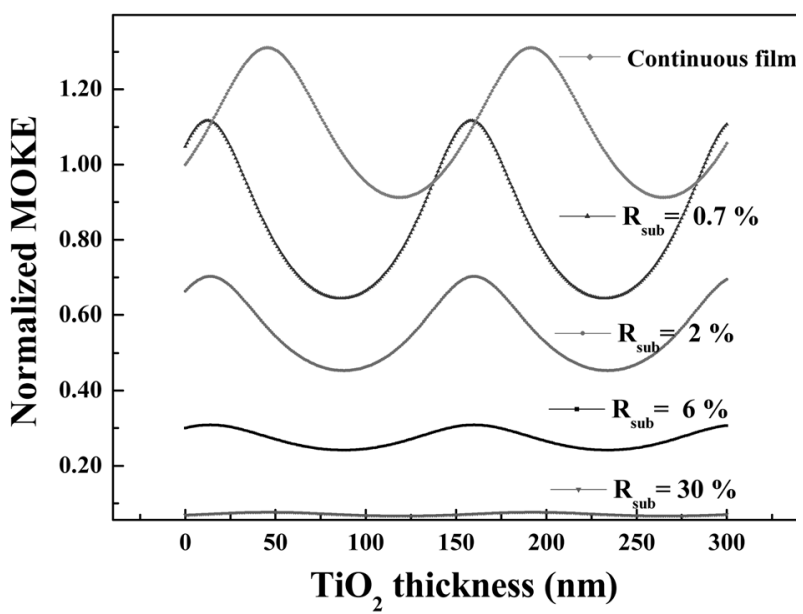

Fig. 4. MOKE signals, normalized by bulk without dielectric layers, for a continuous film and 5 -nm thick and $100-\mathrm{nm}$ wide nanowires on various substrates as a function of the additional dielectric layer $\left(\mathrm{TiO}_{2}\right)$ thickness.

the value was $105 \%$ of its bulk value. Therefore, a welldesigned nanowire with an additional dielectric layer coating on the anti-reflection coated substrate had a large enough signal for the MOKE and could be a useful direction measurement without an average. The second finding was the condition of the maximum MOKE. The period was not changed from the continuous film, but the dielectric layer thickness is changed greatly, indicating the need for detailed calculations in order to achieve maximum enhancement and that the conditions determined from the continuous films cannot be taken at face value. The calculations of $R_{\text {sub }}=6.0$ and $2.1 \%$ also showed intermediate results.

\section{Conclusions}

It was found that the MOKE signal from nanostructures can be enhanced by varying additional dielectric layer thickness and ferromagnetic layer thickness, as well as tailoring substrate reflectivity. The reflected beam can be suppressed from the non-magnetic substrate by employing an anti-reflection coated substrate, leading to MOKE signal enhancement. Furthermore, it was found that the enhancement effect due to the additional dielectric layer was quite different from the continuous film, and that additional cautions are required for MOKE signal optimization in the nanostructures.

\section{Acknowledgments}

This research was supported by the Nano R\&D program through the Korea Science and Engineering Foundation 
funded by the Ministry of Science \& Technology (200802553).

\section{References}

[1] S. O. Demokritov and B. Hillebrands, J. Magn. Magn. Matter. 200, 706 (1999).

[2] L. Guidoni, E. Beaurepaire, and J.-Y. Bigot, Phys. Rev. Lett. 89, 017401 (2002).

[3] D. A. Allwood, G. Xiong, M. D. Cooke, C. C. Faulkner, D. Atkinson, N. Vernier, and R. P. Cowburn, Science 296, 2003 (2002).

[4] G. S. D. Beach, C. Nistor, C. Knutson, M. Tsoi, and J. L. Erskine, Nat. Mat. 4, 741 (2005).

[5] D.-H. Kim and C.-Y. You, J. Magnetics 13, 70 (2008).

[6] Naser Qureshi, Suqin Wang, Mark A. Lowther, Aaron R. Hawkins, Sunghoon Kwon, Alexander Liddle, Jeffrey Bokor, and Holger Schmidt, Nano Lett. 5, 1413 (2005).

[7] Ursula. J. Gibson, Lindsay F. Holiday, Dan A. Allwood, Swaraj Basu, and Paul W. Fry, IEEE Trans. MAG 43, 2740 (2007).

[8] L. F. Holiday and U. J. Gibson, Optics Express 14, 13007 (2006).

[9] D. A. Allwood, G. Xiong, M. D. Cooke, and R. P. Cowburn, J. Phys. D: Appl. Phys. 36, 2175 (2003).

[10] P. R. Cantwell, U. J. Gibson, D. A. Allwood, and H. A. M. Macleod, J. Appl. Phys. 100, 093910 (2006).

[11] S. Wang, A. Barman, H. Schmidt, J. D. Maas, A. R. Hawkins, S. Kwon, B. Harteneck, S. Cabrini, and J.
Bokor, Appl. Phys. Lett. 90, 252504 (2007).

[12] Anjan Barman, Suqin Wang, Jeffrey D. Maas, Aaron R. Hawkins, Sunghoon Kwon, Alexander Liddle, Jeffrey Bokor and Holger Schmidt, Nano Lett. 6, 2939 (2006).

[13] K. Nakamura, T. Asaka, S. Asari, Y. Ota, and A. Itoh, IEEE Trans. MAG 21, 165 (1985).

[14] Kwang-Ho Shin, Masahiko Mizoguchi, and Mitsuteru Inoue J. Magnetics 12, 129 (2007).

[15] M. Mansuripur, The Physical Principles of Magneto-Optical Recording, Cambridge University Press, Cambridge, U.K., (1995) pp. 214-245.

[16] C.-Y. You and S.-C. Shin, J. Appl. Phys. 84, 541 (1998).

[17] C.-Y. You and S.-C. Shin, J. Magn. Magn. Mater. 198, 573 (1999).

[18] J. Zak, E. R. Moog, C. Liu, and S. D. Bader, J. of Magn. Magn. Mater. 89, 107 (1990).

[19] CRC Handbook of Chemistry and Physics, 68th ed. (CRC Press, Boca Raton, 1987-88)

[20] G. S. Krinchik and V. A. Artemev, Sov. Phys. JETP 26, 1080 (1968).

[21] Y. He, T. Kojima, T. Uno, and S. Adachi, IEICE Tran. Elec., E81-C, 1881 (1998).

[22] J. Liu, B. Xu, and T. C. Chong, Jpn. J. Appl. Phys. 39, 687 (2000).

[23] K. Balasubramanian, A. S. Marathay, and H. A. Macleod, Thin Solid Films 164, 391 (1988).

[24] M. Mansuripur, G. A. N. Connell, and J. W. Goodman, J. Appl. Phys. 53, 4485 (1982). 